\title{
Lonza's SSP for Early Phase Delivery
}

\author{
Beat Schmidt*
}

\begin{abstract}
Lonza, the leading custom manufacturer of chemical intermediates and active ingredients as well as biopharmaceuticals for the pharmaceutical and agrochemical industries, has recently expanded into a new state-ofthe-art seven-storey small-scale plant (SSP) whose task is to satisfy both clinical supply and process development with sophisticated and lean cGMP-compliant equipment. With this investment, Lonza has now closed the gap between kilo lab and launch plant in order to provide one-stop shopping for API and drug product developmentthrough-approval.
\end{abstract}

Keywords: cGMP · CMO · Investment · Lonza · Small-scale plant

Lonza offers a full range of top quality services to world-leading pharmaceutical groups including process $\mathrm{R} \& \mathrm{D}$ and analytical methods development. Traditionally Lonza's strength has been - supported by a strong technology platform - optimization and scale-up of processes to industrial production, validation and multi-ton production. Lonza has built up an outstanding reputation through collaborations with big pharmaceutical companies and by providing them with top-quality products in ton lots.

With the changing 'pharmaceutical landscape' the number of pharmaceutical companies has been dramatically reduced by mega mergers leading to pharmaceutical giants. The main driving forces behind this development were cost reduction, improvement of R\&D pipeline and the empowerment of marketing and sales. Another fact with regard to outsourcing activities is that a growing share of new, innovative substances is coming from start-ups who have limited or no manufacturing capabilities.

\footnotetext{
${ }^{*}$ Correspondence: Dr. B. Schmidt

Lonza Ltd

Valais Works

Exclusive Synthesis

SSP E-52

$\mathrm{CH}-3930$ Visp

Tel.: +419486264

Fax: +419476264

E-Mail: beat.schmidt@lonza.com
}

In order to respond appropriately to the changing, more competitive landscape and to keep delivering best service to our customers, Lonza's management have taken the decision in 2001 to invest further in a small-scale production facility, the SSP (small-scale plant) (Fig. 1).

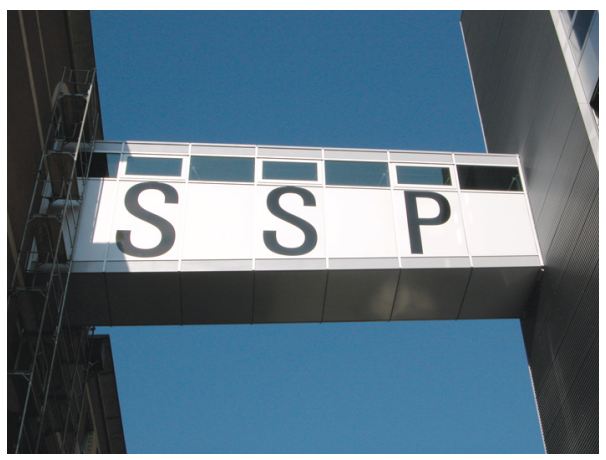

Fig. 1. Gangway SSP connecting the R\&Dbuilding E-38 with SSP tower E-52

\section{Strategic Role of the SSP Plant}

Due to better screening methods the total numbers of pharmaceutical products have increased significantly over the past years. In addition, the complexity of modern drugs has led to a higher average number of chemical steps in synthesis. One measure of our customers to bring down costs has been to reduce the development time for drug candidates from around 10 to 6-7 years. Custom manufacturing organizations (CMO) are more and more forced to jump into the development cycle at a much earlier stage and share the risk with their customer. It is quite difficult and costly to tackle such tasks in a timely fashion with commonly used GMP production facilities. 'Make something new, not with the same degree of difficulty' was Lonza's answer to address appropriately these customer needs in early development stages. The new SSP building was built to close the gap between kilo-lab and launch plant. With this investment Lonza has demonstrated its willingness to provide its customers with a onestop-shop service and support the customers' products throughout their life cycle by sample preparation (kilo lab), small-scale manufacturing (SSP), process development (R\&D), launch quantities (Launch Plant) and large scale manufacturing (FCC, Riverside etc.).

\section{Design of the SSP}

Already at the kick-off meeting, where the newly assembled interdisciplinary Lonza team started their work, it was obvious that we needed a unit, which was independent in terms of its operating systems but which would still have the back-up of the other Lonza services, a newly formed SSP group with a different mindset pertaining to the scaling-up of the process, with a focus on speed in producing the required amount - in most cases with a laborious and non-optimized lab process. Within Lonza's manufacturing capabilities the objectives of the SSP were thus defined:

- To produce small quantities in a speedy manner, which should help to strengthen the business relationship with our key customers

- To be an instrument in generating new business, although the dropout rate will be high 
- To gain better information about the customers' pipeline

- To develop processes which fit into our commercial plant and allow a smooth transfer from small scale to our largescale vessels

- To be a door opener for new customers, who could start business and build up confidence in Lonza with an SSP-project.

For the newly formed team it was an atypical investment project due to the fact that the total investment sum had already received approval from the board based on a vague and sketchy study from our top management. Therefore the investment had to be challenged to a 'design to cost' strategy. The team decided to split the investment in two phases. This approach allowed us to get on stream with the plant earlier and to upgrade in phase II based on the gained teachings and experience made during start-up of phase I.

\section{Speedy Building Activities}

The team discussed the different geographical site options for the SSP within Lonza facilities all over the world. Based on the assessment of different criteria we finally chose Visp in Switzerland. The new SSP building was placed in close proximity to our R\&D and launch plant facilities to profit most from existing synergies (Fig. 2). Thus, technology transfer would be straightforward if a customer decided to continue development and production. The whole building concept was elaborated within two months in close collaboration with an architecture firm located in Basel. We filed the allowance for construction in March 2002. The start of the building activities was in June 2002 and thanks to an amazing performance of the local building contractor we were able to finish the shell of the seven-storey building by the end of 2002. Commissioning and qualification activities were accomplished in the next six months so the SSP could go on stream from mid-year 2003. The SSP building contains six production floors and on top an additional empty floor for additional lab space.

The building is separated into an exzone production area and infrastructure non ex-zone area with auxiliary spaces like stairs, lift, lounge, offices, and storage room. A gangway on floor four connects the SSP building E-52 with the adjacent R\&D building E-38, which is also a symbol for the close collaboration and dependence of the co-workers in these two buildings.

The SSP was built to manufacture key intermediates and APIs according to cGMP guidelines. The production area makes a very tidy impression with well-organized pipelines and clutch and valve systems. We
Fig. 2. SSP tower in close proximity to the adjacent launch plant and R\&D center

Fig. 3. Shift worker operating a typical 160 I SSP reactor

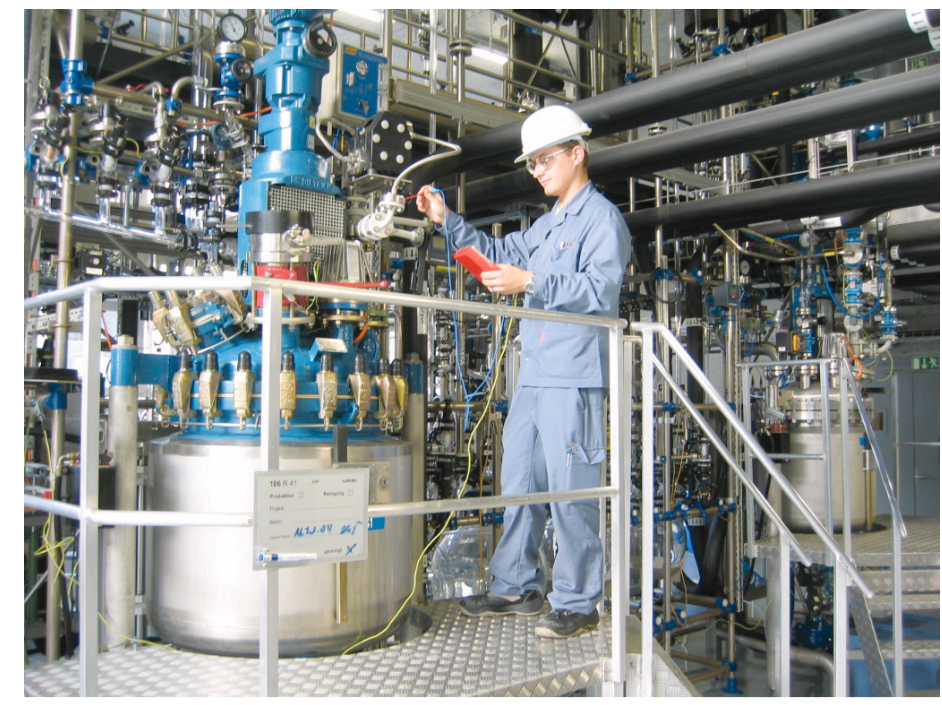

designed a concise reactor set-up, which is highly flexible. It allows us easy cleaning procedures and short changeover time between steps. The SSP is currently equipped with six reactors (160-250 liters), all of them glass-lined or hastelloy, one of which can be used for hydrogenations up to 15 bars and another for cryogenic reactions to $-80{ }^{\circ} \mathrm{C}$ (Fig. 3).

Most of the auxiliary vessels and flasks connected to the reactors are made of glass. Part automation allows staff computer controlled setting with full audit trail and monitoring of basic features like heating/cooling ramps and stirring speed combined with manual operations for additional manipulations. Every reactor is an independent unit where no cross contamination can occur, but according to the needs of the project, we can connect each reactor to any other. Open handling of chemicals in the production area is forbidden and thus all chemicals are transferred in a closed manner. On the separation floor a filter-dryer with a glove box attached, a mobile centrifuge, tray dryers and many mobile filters are avail- able. Several cubicles with air-lock system and controlled-air conditions are in place, where a safe and contamination-free solid handling is guaranteed both for products and personnel. Programming work, hard copies from the computer system and storage of data are done in a separate info room (21CFR part 11 compliant).

Complementary to the SSP the nearby GMP-kilo-lab is equipped with two 301 reactors and numbers of 201 reactors to produce compounds in the lower $\mathrm{kg}$-scale (Fig. 4).

For gram quantities we still have the option to use the GMP clean room lab on top of the R\&D building E-38.

\section{Outlook}

"We should remember that shifting risk to third parties is one of the main reasons why big pharmaceutical groups even started outsourcing" [1]. We believe the SSP is an effective tool to strengthen Lonza's overall position in the increasingly com- 


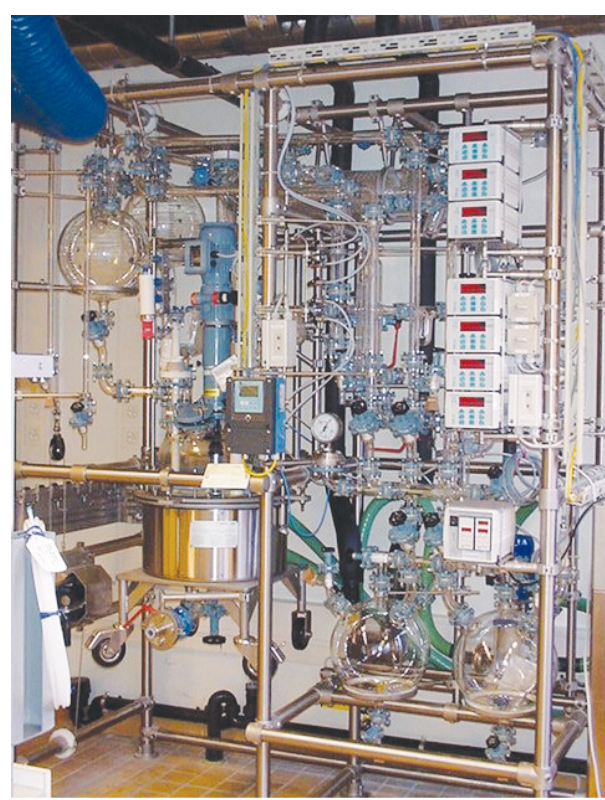

Fig. 4. Kilo lab is equipped with 30 । reactors (Büchi) and several smaller size glass reactors

petitive custom manufacturing arena. We are very pleased with the start-up phase and the obtained utilization figures in the first year. We could also profit from the open feedback from our customers to eliminate initial 'teething problems'. We are now very confident concerning the next upgrade phase, where additional reactors will be placed based on the experience gathered in phase I. As a customer-oriented service company we have learned to respond to

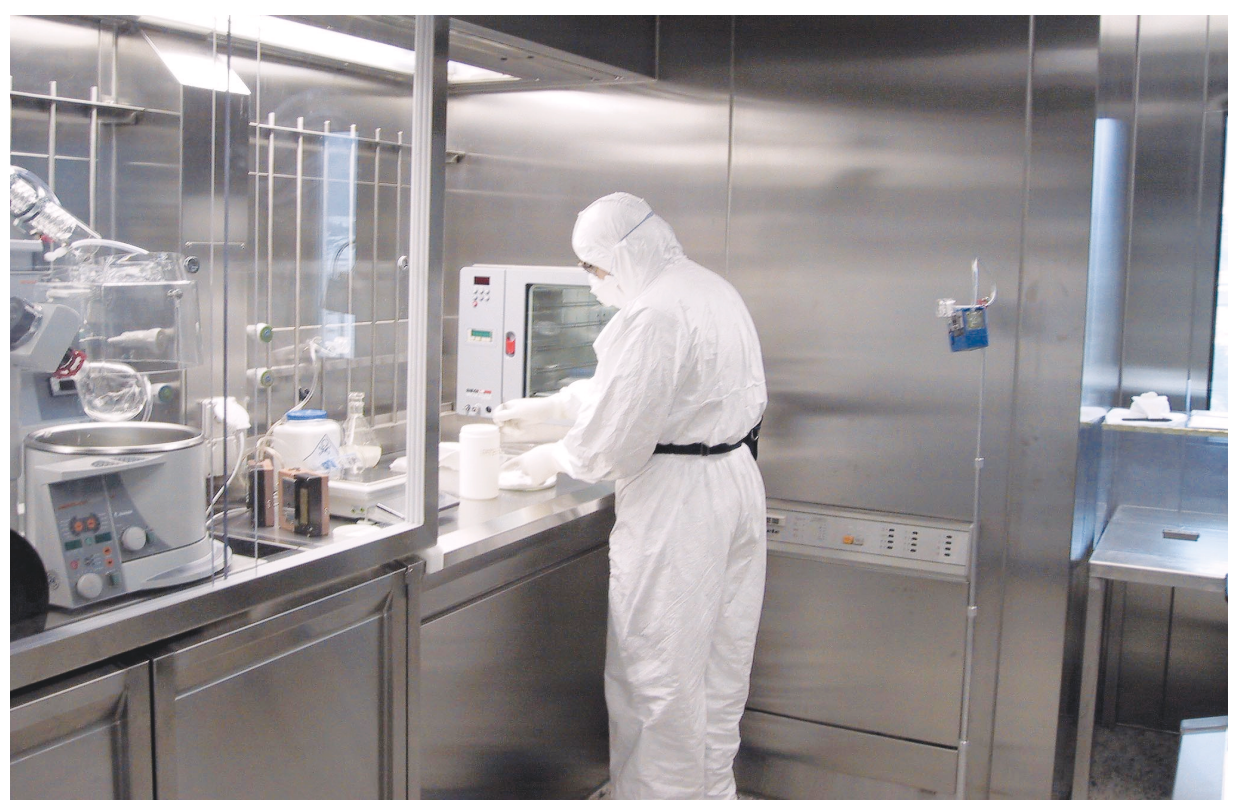

Fig. 5. A fully equipped qualified clean room with air-lock system is available on top of building E-38 for kg-quantities under GMP or also suitable for handling of highly active compounds

customer needs and deliver according to their requirements. Efficient project management and resource planning under extreme time pressure (including $\mathrm{QC}$ ) is a must. We have formed small project teams, which are empowered to handle a project in close collaboration with the customer. The project leader has to allocate the provided resources to reach the timelines. Normally the SSP group with around 30 coworkers works a day shift model. However, people are very motivated and are - in the event of time constraints - also willing to switch to a two or three shift model. Speed, quality and value for the customers, plus the celebration of success in the project team after the campaign are our ultimate goal.

Received: November 19, 2004

[1] E. Mössl, CHIMICA OGGI/Chemistry Today 2001, 44-46. 